\title{
Revealing Cosmic Magnetism with Radio Polarimetry
}

\author{
Bryan M. Gaensler* \\ School of Physics, The University of Sydney \\ E-mail: bgaensler@usyd.edu.au
}

\begin{abstract}
While gravitation sustains the on-going evolution of the cosmos, it is magnetism that breaks gravity's symmetry and that provides the pathway to the non-thermal Universe. By enabling processes such as anisotropic pressure support, particle acceleration, and jet collimation, magnetism has for billions of years regulated the feedback vital for returning matter to the interstellar and intergalactic medium. After reviewing recent results that demonstrate the unique view of magnetic fields provided by radio astronomy, I explain how the Square Kilometre Array will provide data that will reveal what cosmic magnets look like, how they formed, and what role they have played in the evolving Universe.
\end{abstract}

From Planets to Dark Energy: the Modern Radio Universe October 1-5 2007

The University of Manchester, UK

*Australian Research Council Federation Fellow 


\section{Introduction: Magnetism Matters!}

Most astronomical research does not explicitly incorporate the effects of magnetic fields. Nevertheless, there are fundamental reasons why we need to try to understand the various roles played by magnetism in astrophysical processes.

Zweibel [1] provides two specific, clear motivations for further studies of cosmic magnetic fields. First and foremost, the origin of magnetic fields in the Universe is a fundamental and unsolved cosmological problem [2, 3]. We do not know whether the first magnetic fields emerged through exotic processes such as phase transitions or string cosmology, or through standard plasma physics such as turbulence, instabilities or the battery effect. It is unclear whether the diffusion of these seed fields throughout the Universe was then a top-down or a bottom-up process. Were magnetic fields in the early Universe strong enough to moderate the formation of large-scale structure? What role could these fields have played in the formation of the first stars and galaxies?

Second, magnetic fields are the key to answering many long-standing problems in plasma physics and astrophysics [4, 5]. On the largest scales, the coherent magnetic fields that stretch over enormous physical scales in galaxies and clusters allow us to test the extremes of dynamo theory and turbulence. Magnetic fields are at the core of any viable theory for the acceleration and propagation of cosmic rays. Magnetism drives the physics, geometry and evolution of active galactic nuclei (AGN). And finally, a whole variety of important physical processes that drive galactic ecology, including star formation, thermal conduction, diffusion and accretion, all rely heavily on the strength and geometry of the ambient magnetic field.

If our goal as astronomers is to better understand the Universe, then we need to include magnetic fields in our observations and in our models. In this paper, I outline some of the recent progress and future prospects in this area. In $\$ 2$, I briefly review the ways in which radio data can map magnetic fields out to large distances. In $\$ 3$ \& $\$ 母$, I show some recent applications to the largescale magnetic fields of the Milky Way and of the Large Magellanic Cloud (LMC), respectively. In $\$$, I discuss some of the experiments on magnetic fields at intermediate and high redshifts that can be conducted with the Square Kilometre Array (SKA).

\section{Mapping Magnetic Fields}

There are various indirect approaches to studying magnetic fields in astrophysical sources [6]. Measurements of optical starlight polarisation (e.g., [7]), polarised radio synchrotron emission (e.g., [8]) and infrared/sub-mm dust polarisation (e.g., [9]) all characterise the structure of the magnetic field in the plane of the sky, $B_{\perp}$. While this provides information on two of the three spatial components of the field vector, a crucial limitation of these approaches is that they all only provide the orientation of $B$, but not its direction. For example, simple compression of a tangled magnetic field can produce a set of polarisation vectors which will appear quite ordered, but which have no spatial coherence.

Zeeman splitting is distinct from these other approaches, in that it measures the line-of-sight component of the magnetic field $\left(B_{\|}\right)$, and can determine the direction of this field (e.g., [10, 11]). However, most Zeeman experiments require long integrations, and often probe localised regions of relatively high gas density. 
The remaining approach to studying magnetic fields is Faraday rotation, which has proven to be a very powerful probe of $B_{\|}$. Faraday rotation is a change in the linear polarisation angle of a radio signal as it propagates through a magnetised plasma. The orientation of the observed electric field vector is:

$$
\Theta=\Theta_{0}+\mathrm{RM} \lambda^{2}
$$

where $\lambda$ is the observing wavelength, $\Theta_{0}$ is the intrinsic polarisation angle emitted by the source, and $\Theta$ is the observed polarisation angle. The rotation measure (RM) is a path integral through the magneto-ionised foreground:

$$
\mathrm{RM}=K \int n_{e} B_{\|} d l
$$

where the free electron density, $n_{e}$, and line-of-sight field strength, $B_{\|}$, are integrated from the observer to the source along a line element $d l$, and $K$ is a constant. An example of Faraday rotation seen in the polarised emission from a radio pulsar is shown in Figure 1.

Faraday rotation has three important advantages. First, as for Zeeman splitting, it provides a direction for $B_{\|}$along a given sightline, allowing us to test for field coherence if we have RM data for several adjacent positions on the sky. Second, since Faraday effects are strongest in the radio part of the spectrum, interstellar extinction can be disregarded, and magnetic fields can thus be probed out to cosmological distances. Finally, Faraday rotation is an "absorption" experiment, in the sense that the signal-to-noise ratio of the measurement is determined by the flux of the polarised background source, rather than any properties of the region being studied. Measurements of magnetic fields in regions that are not easily observable directly, such as the Galactic halo and the

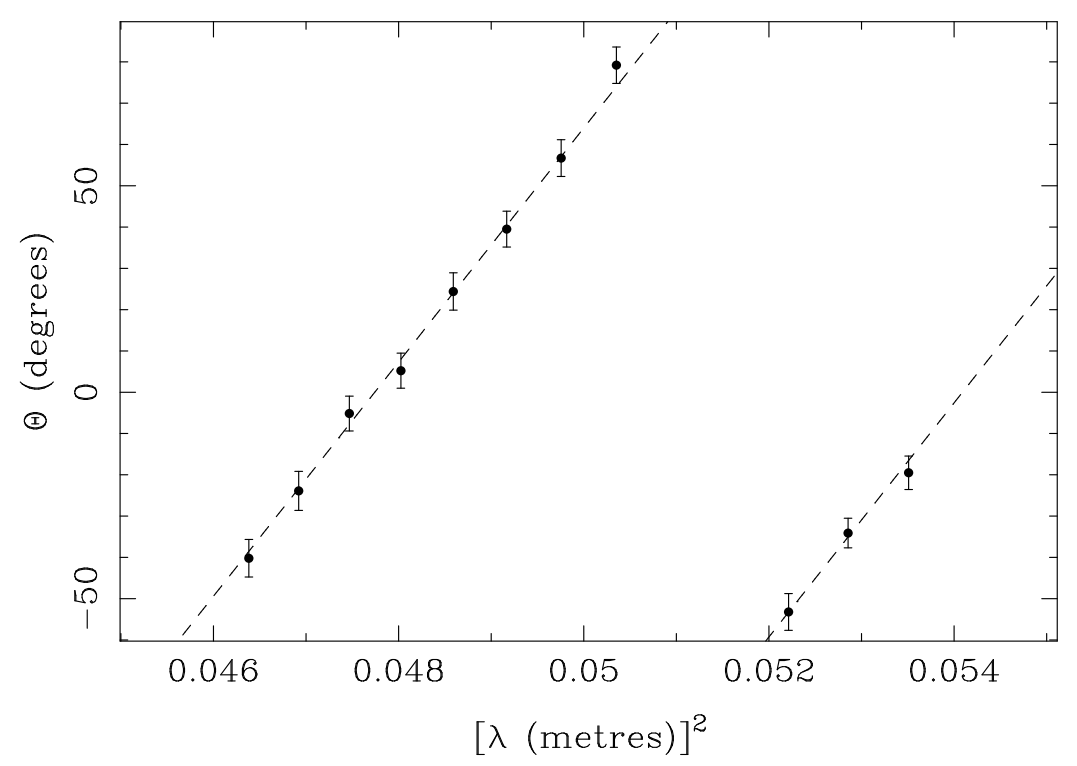

Figure 1: Faraday rotation in the Galactic interstellar medium, seen toward the radio pulsar B1154-62 [12]. The data points indicate multi-channel spectropolarimetric measurements in the $20 \mathrm{~cm}$ band made using the Australia Telescope Compact Array (ATCA), while the dashed line shows the best fit of Equation 2.1 to these data. The slope of this line is $\mathrm{RM}=+495 \pm 6 \mathrm{rad} \mathrm{m}^{-2}$, indicating that the mean magnetic field along this sightline is directed toward us. 
intergalactic medium (IGM), then become possible, provided that appropriate background sources can be identified.

\section{The Galactic Magnetic Field}

It has long been established that there are magnetic fields of significant strength throughout the interstellar medium of the Milky Way [13, 14]. Points of general consensus are that the field has both large-scale (ordered) and small-scale (random) components, that the magnetic field is concentrated in and is generally oriented parallel to the disk of the Galaxy, and that the ordered component of the field probably broadly follows the spiral arms. Studies of pulsar RMs, extragalactic RMs and optical starlight polarisation show that the large-scale field within a kpc of the sun is directed clockwise (as viewed from the North Galactic Pole) [7], 15], but that the field in the SagittariusCarina spiral arm, a few kpc closer to the Galactic Centre, is oriented counterclockwise [16, 17]. This clearly indicates that a large-scale reversal of the large-scale magnetic field occurs somewhere between these two arms. The presence and properties of such reversals provide crucial constraints on dynamo theories and on the origin of galactic magnetic fields (see [18]).

For more distant parts of the Galaxy, especially beyond the Galactic Centre, the geometry of the field is still unclear, mainly due to the lack of known pulsars in these regions. Recent studies have come to a variety of conclusions as to the overall Galactic field geometry. Just to provide a few examples: Weisberg et al. [19] have argued that the field follows the spiral arms, with a reversal between each arm; Han et al. [20] have proposed that the field is a spiral, but with reversals on both sides of every spiral arm, so that fields in arm and inter-arm regions are directed in opposite directions; Vallée [21] has suggested that the field is purely azimuthal rather than a spiral, with reversals in concentric rings. As noted by Vallée [22], the situation is not unlike the early exploration of Australia, during which half the coastline had been mapped in detail, with the more distant parts trailing off into "terra incognita", as shown in Figure 2 .

\subsection{Polarised Extragalactic Sources}

Most of the work mentioned above has relied heavily on pulsar RMs. Pulsars have several advantages for such studies: they are at known distances (albeit with significant uncertainty for individual sources), they are within the Galaxy (so that pulsars at a range of distances can be used to make differential magnetic field measurements), and both dispersion and Faraday rotation of their signals can be measured (so that the $n_{e} d l$ term in Eqn. [2.2]) can be independently estimated).

However, pulsars also have their limitations. Most pulsars are relatively nearby. This means that individual $\mathrm{H}$ II regions and other dense gas clumps along the line of sight can make a large contribution to the overall RM, making it more difficult to probe the properties of large-scale magnetic fields (e.g., [24]). The distance estimates to individual pulsars from standard models (e.g., [25]) can sometimes be significantly in error, making it difficult to match the magnetic field direction inferred from a pulsar's RM to Galactic structure such as spiral arms. Pulsars are a small population of faint sources (there are less than 2000 known pulsars, with a median $1.4 \mathrm{GHz}$ flux of $\sim 0.5 \mathrm{mJy}$ ), so that the density and total number of RM sightlines that they can provide are both relatively low. Finally, even in regions such as along the Galactic plane, where the sky density of pulsar RMs is 


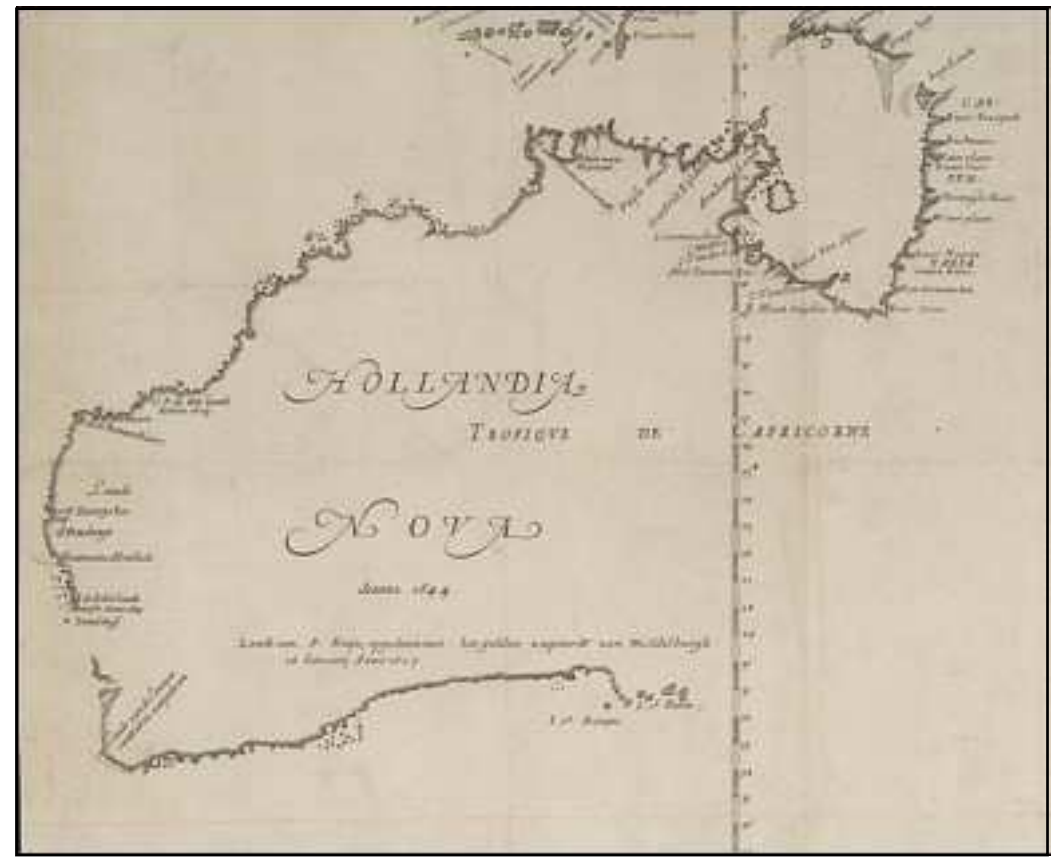

Figure 2: An early map of Australia, drawn by the French cartographer Melchisédech Thévenot ("Hollandia Nova detecta 1644; Terre Australe decouuerte l'an 1644", Map RM 689A, [23]). Reproduced with permission of the National Library of Australia.

higher than average, the RM sample cannot be easily smoothed to bring out large-scale structure, because each pulsar is at a different distance.

To make substantial further progress in studying the Milky Way's magnetism, an additional approach is therefore needed. We have consequently undertaken a coordinated effort to greatly expand the number of polarisation and RM measurements for extragalactic sources (i.e., radio galaxies and AGN). These sources are at large distances, and so provide a measurement of the Faraday rotation through the entire Milky Way along a particular direction. In fact, the observed RM is the sum of multiple contributions: from the source itself, from the IGM, from the Earth's ionosphere, and from the Milky Way. However, in most cases, and certainly when averaged over a large sample of such data, the first three terms are small and can be disregarded, and the total RM can be used as a useful probe of the Galaxy's magnetism.

It is important to emphasise that RM data for extragalactic sources only provides an integral of $n_{e} B_{\|} d l$, as per Equation (2.2). Thus if the actual value of $B_{\|}$is of interest, a model is needed of $n_{e}$ as a function of $l$ (e.g., [25]). However, many important problems require knowledge only of the geometry of $B$, without accurate estimates of its strength. Since the sign of the RM is determined by the sign of $B_{\|}$, the overall magnetic configuration can be studied from the RMs alone, without needing to invert the integral.

Brown et al. [26, 27] have recently completed two large surveys of extragalactic RMs behind the Galactic plane: 380 RMs in the outer Galaxy, derived from the Canadian Galactic Plane Survey $(\mathrm{CGPS})^{1}$ and $148 \mathrm{RMs}$ in the inner Galaxy, measured as part of the Southern Galactic Plane Survey

http://www.ras.ucalgary.ca/CGPS/ 

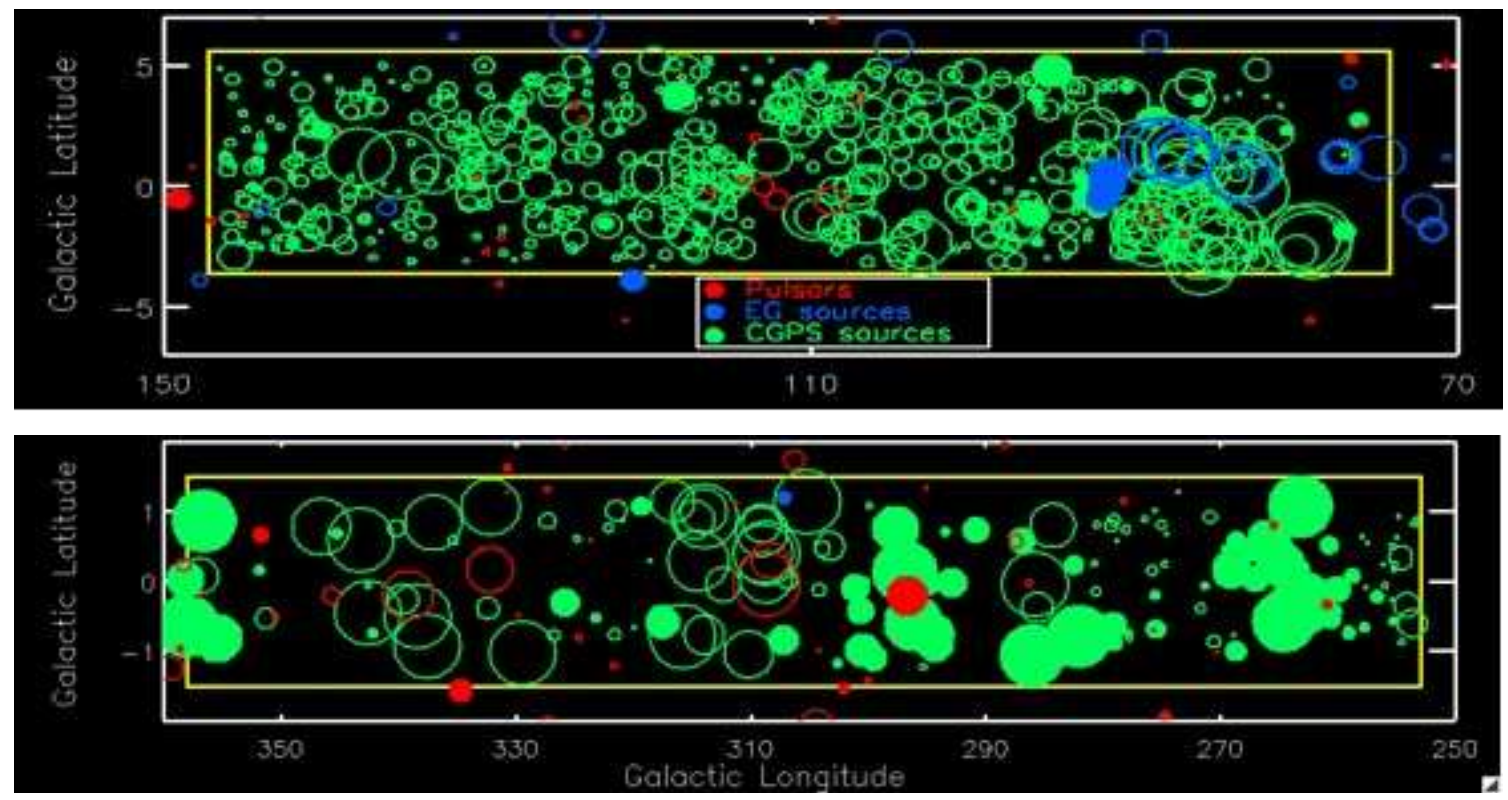

Figure 3: Distribution of RMs in two strips along the Galactic plane, corresponding to the survey regions of the CGPS (upper panel) and SGPS (lower panel) [26, 27]. The circles mark the positions of RM measurements: blue for previously measured extragalactic RMs, red for pulsar RMs, and green for new CGPS/SGPS data. The radius of each circle corresponds to the magnitude of the RM for that source; filled circles indicate positive RMs and open circles show negative RMs. Figures are courtesy of Jo-Anne Brown.

(SGPS). ${ }^{2}$ The results are shown in Figure 3; the vast improvement in the sample size compared to previous data-sets reveals a striking coherence in the large-scale magnetic field configuration. For the CGPS, the RMs are almost all negative, showing that the Perseus spiral arm (through which these sources are viewed) has a clockwise magnetic field [28]. For the SGPS, the situation is considerably more complicated, with alternating regions of positive and negative RMs, with regions of low $|\mathrm{RM}|$ between them. The implications of the RM structure seen in the SGPS are considered in the following section.

\subsection{The Magnetic Geometry of the Milky Way}

Figure 4 shows a smoothed version of the extragalactic RM data from the lower panel of Figure 3 . The spatial coherence of Faraday rotation as a function of Galactic longitude is very clear. There are local maxima of $|\mathrm{RM}|$ in three directions: $\ell \approx 292^{\circ}, \ell \approx 312^{\circ}$ and $\ell \approx 338^{\circ}$, as shown by the dashed vertical lines in Figure $\emptyset$. These sightlines are tangent to the Carina, Crux and Norma spiral arms, respectively. On either side of these peaks are directions where $|\mathrm{RM}| \approx 0 \mathrm{rad} \mathrm{m}^{-2}$, shown by dotted lines in Figure 4 . Superimposed on this oscillatory behaviour, is an overall largescale signature: for $\ell \lesssim 304^{\circ}$, RMs are almost all positive, and for $\ell \gtrsim 304^{\circ}$, RMs are predominantly negative (apart from some high RMs close to the Galactic Centre). This dividing line is marked in red in Figure母.

Qualitatively, these patterns in RM immediately reveals some properties of the global magnetic field structure of the Galaxy. The peaks in $|\mathrm{RM}|$ along each spiral arm demonstrate that the field

2 http://www.atnf.csiro.au/research/HI/sgps 


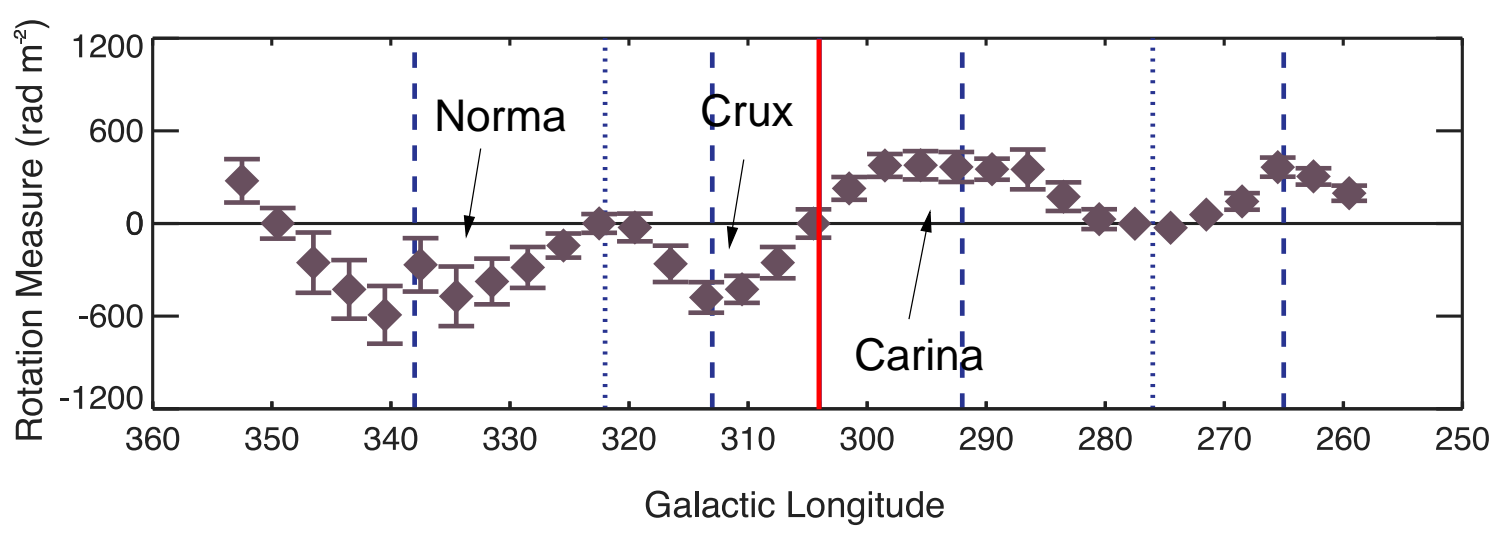

Figure 4: RM vs. Galactic longitude for extragalactic RMs in the SGPS region. The purple diamonds represent smoothed RM data, with error bars indicating the standard error of the mean in each bin. The dotted (dashed) lines indicate approximate longitudes of minimum (maximum) $|\mathrm{RM}|$ in the smoothed distribution. The red line marks the approximate transition from predominantly positive RMs $\left(\ell \lesssim 304^{\circ}\right)$ to predominantly negative $\mathrm{RMs}\left(\ell \gtrsim 304^{\circ}\right)$. Adapted from [27].

strength and/or gas density are high in these regions, while the low values of $|\mathrm{RM}|$ indicate low values of these quantities in the inter-arms (see [27] for a detailed discussion). The change in overall sign of $\mathrm{RM}$ at $\ell \approx 304^{\circ}$ suggests the presence of a large scale reversal in the field along this sightline. of this direction.

This is quantified in Figure 5, where we show a joint fit to extragalactic and pulsar RMs in the southern Galaxy, allowing a series of concentric, spiral, annuli to each have a differing field strength and field direction. The best fit shows that the Galactic magnetic field is primarily clockwise, except for a strong counterclockwise field in the Scutum-Crux spiral arm (and possibly also in the molecular ring in the inner Galaxy). Sightlines at $\ell>304^{\circ}$ pass through the ScutumCrux arm, and are dominated by negative RMs; at $\ell<304^{\circ}$, field lines are directed toward the observer, and RMs are consequently positive. The quality of the fit is indicated in Figure 6, where we compare RM data to the predictions of the model in Figure 5. For extragalactic data, the model RMs and the data match very well. For pulsars, the scatter is larger (mainly because pulsar RM data cannot be meaningfully smoothed), but the major features are reproduced.

This global fit is at odds with earlier studies utilising smaller data-sets, in that it suggests that the Galaxy can be modelled with a predominantly clockwise field, plus a single reversed region. This structure is in line with what is seen also for other spiral galaxies, but needs to be verified by a better mapping of extragalactic RMs in the first Galactic quadrant.

\section{The Magnetic Field of the Large Magellanic Cloud}

The LMC is also particularly amenable to extragalactic RMs as a probe of its magnetic field, because of its large angular extent $\left(\sim 6^{\circ}\right)$ on the sky. Gaensler et al. [29] re-analysed archival LMC data taken with the ATCA, and extracted polarisation and RMs for 292 background sources. The results, shown in Figure ₹, show that RMs are generally positive on the eastern half of the galaxy, and negative on the western half. Analysed in more detail, these RMs reveal a sinusoidal pattern 


\section{Modeled IBI Contours}

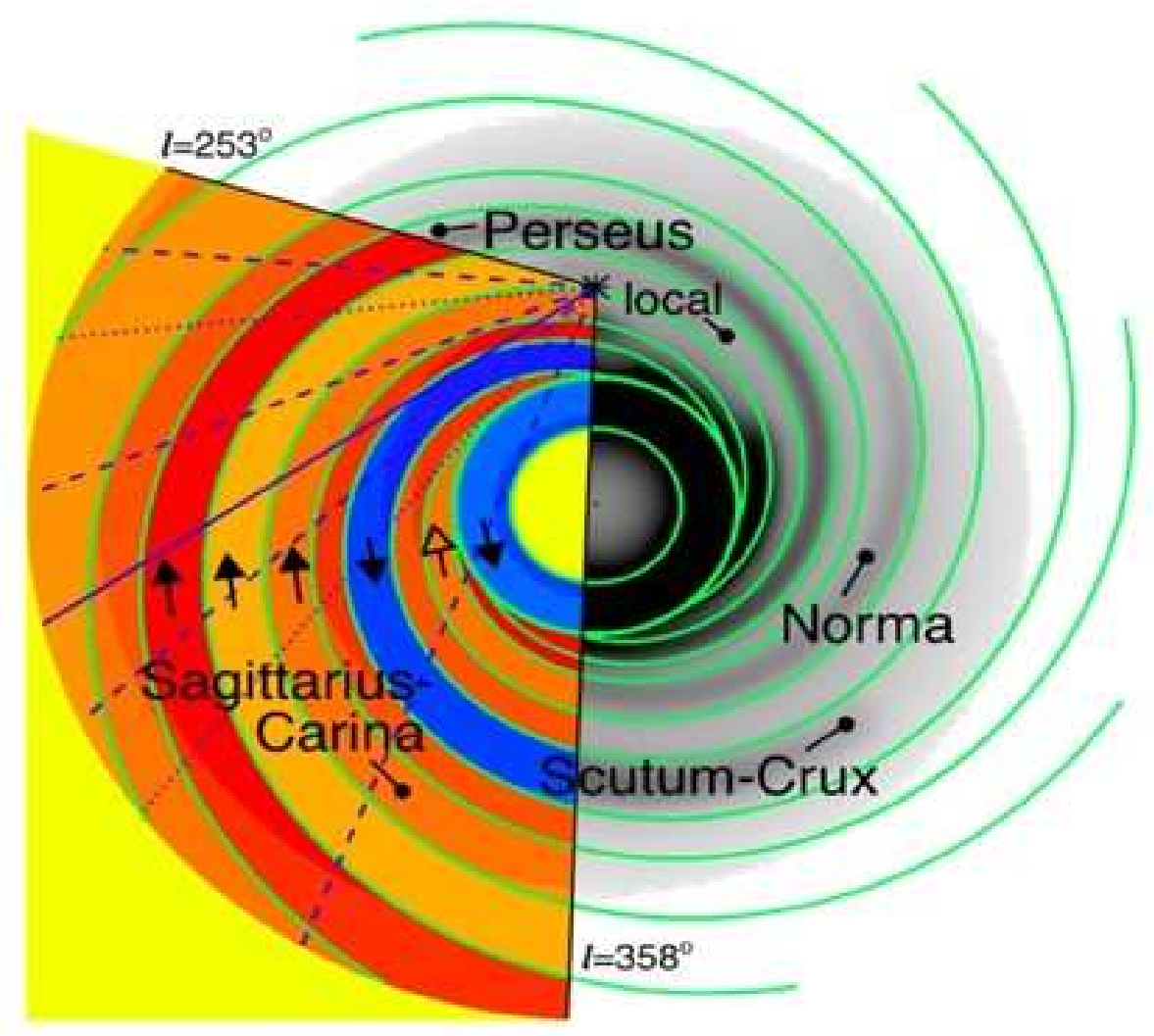

Legend $(\mu \mathrm{G})$

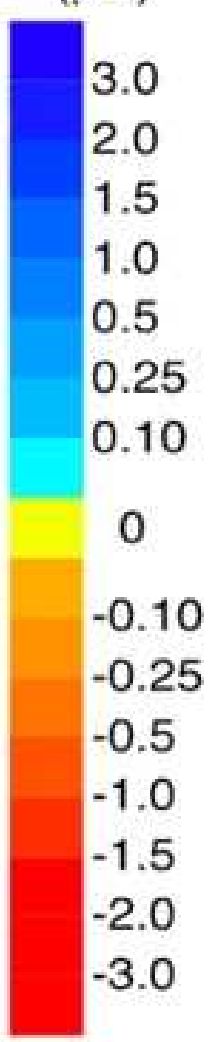

Figure 5: A simple model of the magnetic field in the southern Galaxy, from a joint fit to the RMs of 149 extragalactic sources and 120 pulsars. Coloured regions show the total strength of the model magnetic field in separate regions bounded by the green lines. The limiting longitudes of the SGPS are shown as black lines, while the grey scale represents the NE2001 electron density model of Cordes \& Lazio [25]. Adapted from [27].

as a function of azimuth, implying a coherent, spiral, pattern in the LMC's magnetic field, with a strength of about $1 \mu \mathrm{G}[29]$.

The presence of this relatively strong, ordered, field is somewhat surprising in the LMC. Standard turbulent dynamo theory requires 5-10 Gyr to amplify a weak primordial seed field to microgauss levels, but the repeated tidal interactions between the LMC, Milky Way and Small Magellanic Cloud should disrupt any field that might be slowly built up through this process. The coherent field revealed in Figure 7 must have been amplified and organised rapidly, in only a few hundred million years. One possibility is a cosmic ray dynamo (e.g., [30]), which should thrive in the vigorous starburst environment supplied by the LMC. 


\section{Magnetism with the Square Kilometre Array}

\subsection{The Rotation Measure Grid}

The results presented in $\$ 3$ \& $\$$ 的 can be greatly expanded upon with a larger sample of RMs (see also Kronberg, these proceedings). With the SKA, we envisage an all-sky "rotation measure grid" [31, 32], which would be derived from a 1.4 GHz full-Stokes continuum survey. For an SKA field of view of $5 \mathrm{deg}^{2}$, six months of observing would result in an RMS sensitivity of $\approx 0.1 \mu \mathrm{Jy}$ beam $^{-1}$. To estimate the yield of the resulting RM grid, one needs to consider " $\log N-\log P$ ", i.e., the differential source counts in linear polarisation, analogous the usual $\log N-\log S$ function in total intensity [31, 33]. This results in a distribution like that shown in Figure 8. While there are uncertainties in extrapolating to the low flux levels expected for the SKA (see discussion by [3] ), we can roughly predict that the RM grid should yield about $\sim 10^{8}$ sightlines, with a typical spacing between measurements of $\sim 1^{\prime}$. A simulation of the polarised sky as might be seen with the SKA is shown in Figure 9. Such a data-base will provide a fantastic probe of all manner of extended foreground sources, either individually (like the case of the LMC) or as a statistical ensemble (see [34] and Arshakian et al., these proceedings).

\subsection{The Magnetic Universe}

One of the main applications of the SKA's RM grid will be to study the growth of galactic-

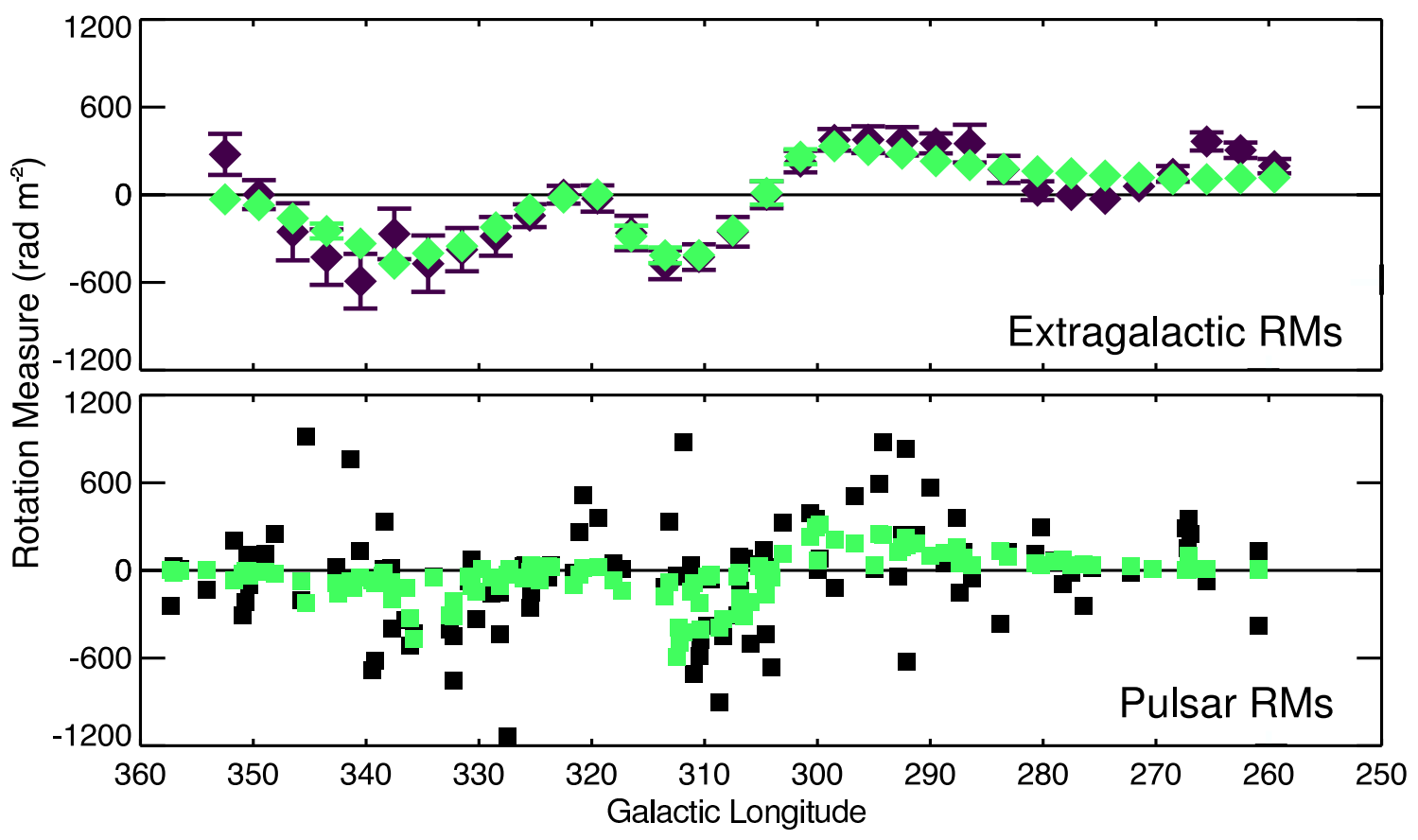

Figure 6: RM vs. Galactic longitude for extragalactic sources (upper; purple points) and pulsars (lower; black points) in the SGPS region; the extragalactic data have been smoothed as in Fig. 7 . The green symbols show the RMs predicted by the best-fit model of [27] at the positions of each of the observed sources. The model data for extragalactic sources have been smoothed in the same way as for the observations. Adapted from [27]. 


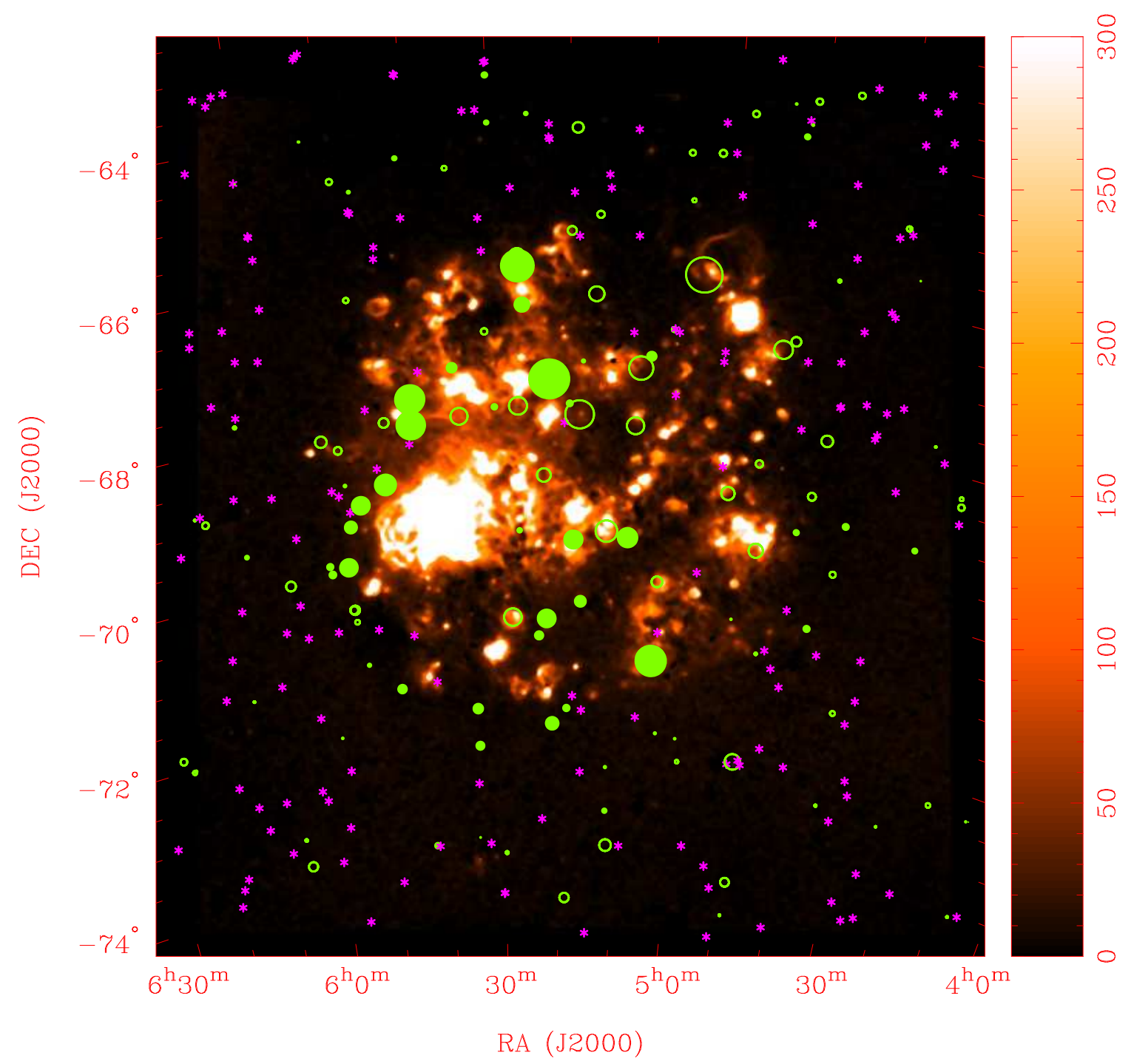

Figure 7: RMs of extragalactic sources behind the Large Magellanic Cloud [29]. The image shows the distribution of emission measure toward the LMC in units of $\mathrm{pc} \mathrm{cm}^{-6}$. The symbols show the position, sign and magnitude of extragalactic RMs: filled (open) circles correspond to positive (negative) RMs, while asterisks indicate RMs which are consistent with zero within their errors. The diameter of each circle is proportional to the magnitude of the RM.

scale magnetic fields as a function of cosmic time. The expectation is that the sightlines to distant extragalactic sources should generally intersect one or more foreground galaxies, as is seen in the Ly- $\alpha$ and Mg II absorption lines in the optical spectra of quasars. Such intervenors should generate an RM signature in the background source, and this signature should potentially evolve with redshift. In particular, Equation (2.2), if rewritten to take into account cosmological effects, contains a $(1+z)^{-2}$ dilution term because of redshift of the emitted radiation, but in some models can also contain co-moving terms with dependencies $n_{e} \propto(1+z)^{3}$ and $B_{\|} \propto(1+z)^{2}$ [河]. The overall RM may then potentially evolve as rapidly as $\mathrm{RM} \propto(1+z)^{3}$, in which case we expect that filaments and absorbers should begin to show an increasingly large RM at higher $z$. If we can obtain 


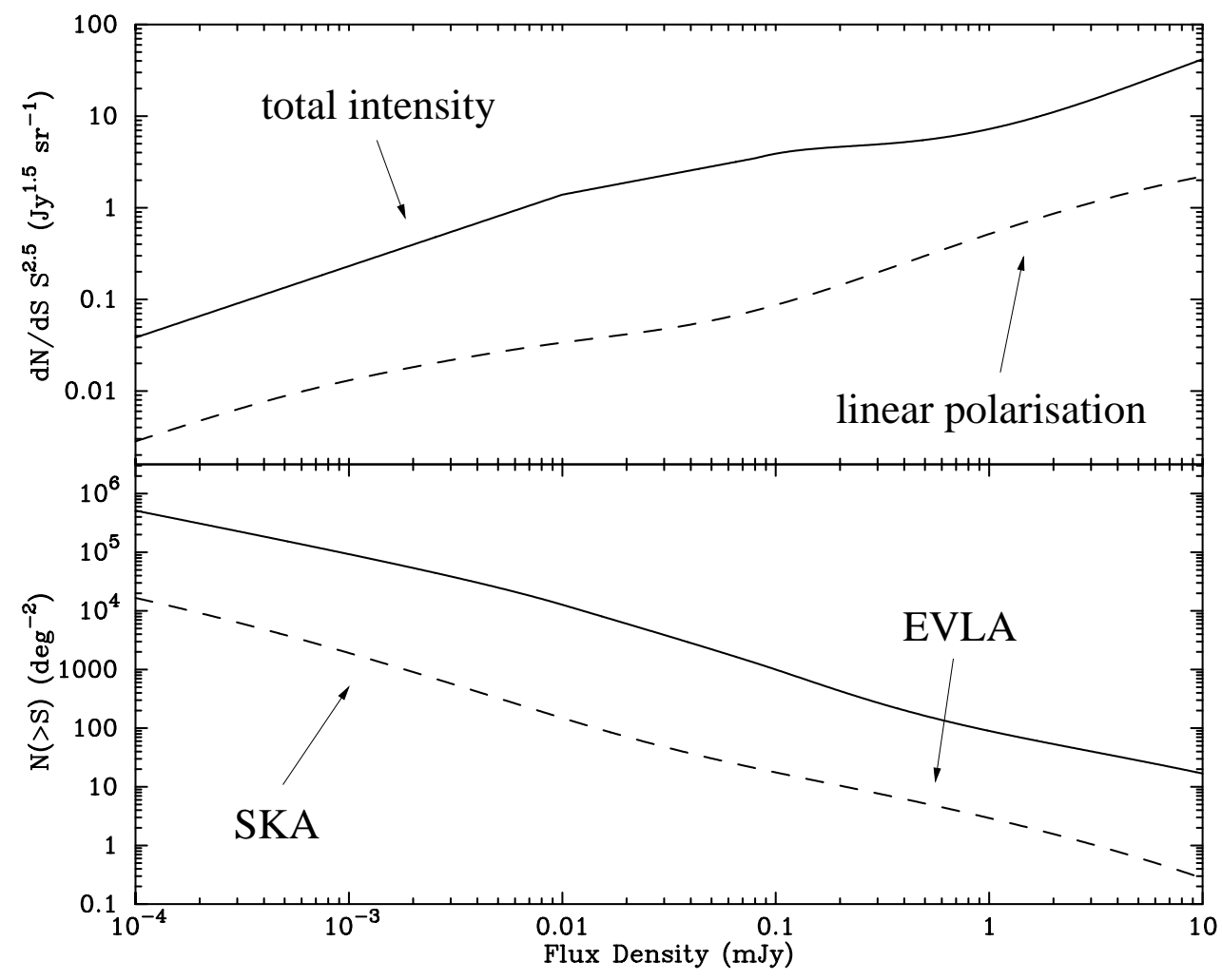

Figure 8: The predicted flux distribution of extragalactic radio sources in both total intensity (solid lines) and in linear polarisation (dashed lines), adapted from [31]. The upper panel shows the differential source count distribution, while the lower panel shows the integral distribution. Approximate flux limits for wide-field surveys with the EVLA and with the SKA are indicated.

a large sample of both RMs (from the SKA) and accompanying redshifts (from the next generation of photometric and spectroscopic optical surveys), we can apply a variety of statistical tests to the distribution of RM vs. $z$ to map the magnetic evolution of the Universe to $z \sim 3$ [35, 36, 37].

\subsection{Magnetic Fields at $z>5$}

There is already good evidence that microgauss-strength magnetic fields exist out to redshifts $z \sim 1-2$ [37, 38]. If we can extend such data out to $z \gtrsim 5$, we can potentially obtain strong constraints on how large-scale magnetic fields were created and then amplified. Such measurements can be made by obtaining RMs for polarised sources at very high redshifts.

Indeed, radio emission has already been detected from two classes of sources at $z>6$ : gammaray burst afterglows [39] and quasars [40]. We currently lack the sensitivity to detect linear polarisation and RMs from these objects, but such measurements should be possible with the SKA. Furthermore, since the cosmic microwave background is linearly polarised, deep observations at the upper end of the SKA frequency range may be able to measure RMs against it [41, 42]. Such an experiment, while challenging, would probe the integrated Faraday rotation over almost all of 


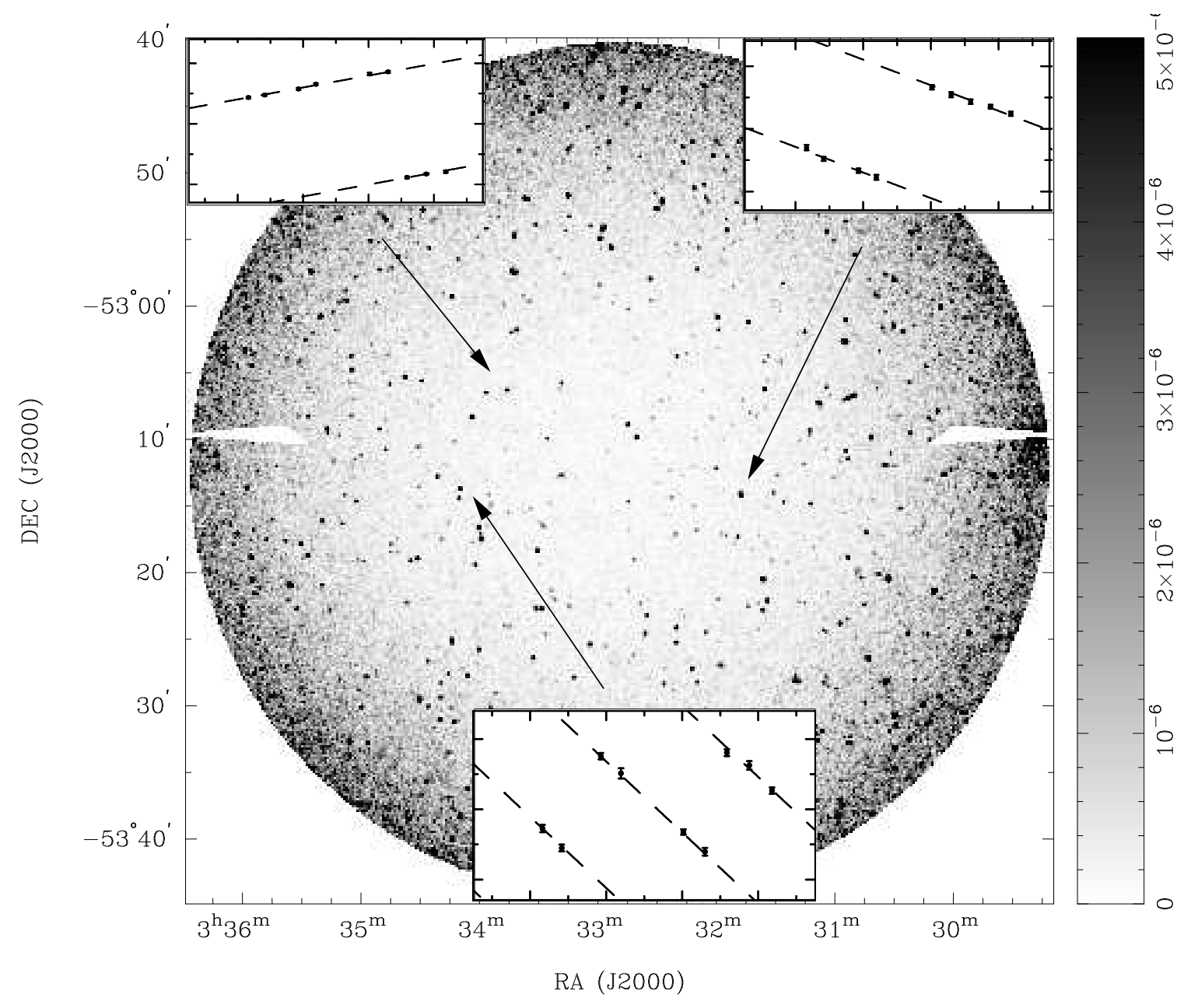

Figure 9: A simulation of a one-hour SKA observation in linear polarisation, for a field of view of $1 \mathrm{deg}^{2}$. The angular resolution is a few arcsec, and the gray scale is in units of $\mu \mathrm{Jy}$. The image was created by squaring the intensity values of an NVSS field to generate a Ricean noise distribution, and then adjusting the flux and spatial scales to simulate the SKA source density predicted by Fig. 8. The insets show some hypothesised distributions of position angle vs. $\lambda^{2}$ for three sources in the field, from which RMs can then be calculated.

the Universe's history. In considering such measurements, it is important to note that an RM measurement to a high- $z$ source does not provide any direct constraints on high- $z$ magnetic fields on its own, since the observed RM will also contain contributions from low- $z$ components of the sightline, and from the Milky Way foreground. Once a high- $z$ RM data point has been obtained, deep radio and optical observations of that field can yield a large number of RMs and redshifts for adjacent foreground objects (see Fig. 9). When the corresponding foreground contribution is then removed, the high- $z$ magnetic field can be isolated studied.

\section{Conclusions}

Cosmic magnetism is a vigorous and rapidly developing field. What makes this area partic- 
ularly relevant for the SKA is that magnetic fields at cosmological distances are uniquely probed at radio wavelengths. By studying the evolution of magnetic fields over the Universe's history, we can simultaneously address a variety of major topics in fundamental physics and astrophysics. This work also has strong synergies with other astronomy and astroparticle experiments, such as Planck, LSST, JWST, HESS and Auger [43, 44.

In the coming years, a host of SKA pathfinders will begin to finally reveal the depth and detail of the polarised sky [45, 46], culminating in an exploration of the full Magnetic Universe with the Square Kilometre Array.

\section{Acknowledgments}

I thank my various collaborators for their contributions to the work reported here, in particular Jo-Anne Brown for providing the material for several figures. This work has been supported by the Australian Research Council (grant FF0561298) and by the National Science Foundation (grant AST-0307358).

\section{References}

[1] E. G. Zweibel, Evolution of magnetic fields at high redshift, Astron. Nach. 327 (2006) 505-509.

[2] D. Grasso and H. R. Rubinstein, Magnetic fields in the early universe, Phys. Rep. 348 (2001) 163-266.

[3] L. M. Widrow, Origin of galactic and extragalactic magnetic fields, Reviews of Modern Physics 74 (2002) 775-823.

[4] D. B. Melrose, Plasma Astrophysics: Nonthermal Processes in Diffuse Magnetized Plasmas. Gordon \& Breach, New York, 1980.

[5] E. M. de Gouveia Dal Pino, G. Lugones and A. Lazarian, Magnetic Fields in the Universe. American Institute of Physics, Melville, New York, 2005.

[6] E. G. Zweibel and C. Heiles, Magnetic fields in galaxies and beyond., Nature 385 (1997) 131-136.

[7] C. Heiles, The local direction and curvature of the galactic magnetic field derived from starlight observations, ApJ 462 (1996) 316-325.

[8] R. Beck and P. Hoernes, Magnetic spiral arms in the galaxy NGC 6946., Nature 379 (1996) 47-49.

[9] D. Ward-Thompson, J. M. Kirk, R. M. Crutcher, J. S. Greaves, W. S. Holland and P. André, First Observations of the Magnetic Field Geometry in Prestellar Cores, ApJ 537 (2000) L135-L138.

[10] T. H. Troland and C. Heiles, Interstellar magnetic field strengths and gas densities: Observational and theoretical perspectives, ApJ 301 (1986) 339-345.

[11] M. J. Reid and E. M. Silverstein, OH masers and the Galactic magnetic field, ApJ 361 (1990) 483-486.

[12] B. M. Gaensler, R. N. Manchester and A. J. Green, Radio continuum and HI observations of supernova remnant G296.8-00.3, MNRAS 296 (1998) 813-823.

[13] E. Fermi, On the origin of the cosmic radiation, Phys. Rev. 75 (1949) 1169-1174.

[14] L. Davis Jr. and J. L. Greenstein, The polarization of starlight by interstellar dust particles in a galactic magnetic field, Phys. Rev. 75 (1949) 1605. 
[15] R. N. Manchester, Pulsar rotation and dispersion measures and the galactic magnetic field, ApJ 172 (1972) 43-52.

[16] M. Simard-Normandin and P. P. Kronberg, Rotation measures and the Galactic magnetic field, ApJ 242 (1980) 74-94.

[17] R. C. Thomson and A. H. Nelson, The interpretation of pulsar rotation measures and the magnetic field of the Galaxy, MNRAS 191 (1980) 863-870.

[18] A. Shukurov, Mesoscale magnetic structures in spiral galaxies, in Cosmic Magnetic Fields (R. Wielebinski and R. Beck, eds.), (Springer, Berlin), pp. 113-135, 2005.

[19] J. M. Weisberg, J. M. Cordes, B. Kuan, K. E. Devine, J. T. Green and D. C. Backer, Arecibo $430 \mathrm{MHz}$ pulsar polarimetry: Faraday rotation measures and morphological classifications, ApJS 150 (2004) 317-341.

[20] J. L. Han, R. N. Manchester, A. G. Lyne, G. J. Qiao and W. van Straten, Pulsar rotation measures and the large-scale structure of Galactic magnetic field, ApJ 642 (2006) 868-881.

[21] J. P. Vallée, Pulsar-based Galactic magnetic map: A large-scale clockwise magnetic field with an anticlockwise annulus, ApJ 619 (2005) 297-305.

[22] J. P. Valleee, Metastudy of the spiral structure of our home Galaxy, ApJ 566 (2002) 261-266.

[23] M. Thévenot, Relations de Divers Voyages Curieux. J. Langlois, Paris, 1663.

[24] D. Mitra, R. Wielebinski, M. Kramer and A. Jessner, The effect of HII regions on rotation measure of pulsars, A\&A 398 (2003) 993-1005.

[25] J. M. Cordes and T. J. W. Lazio, NE2001. I. A New Model for the Galactic Distribution of Free Electrons and its Fluctuations, preprint (arXiv:astro-ph/0207156).

[26] J. C. Brown, A. R. Taylor and B. J. Jackel, Rotation Measures of Compact Sources in the Canadian Galactic Plane Survey, ApJS 145 (2003) 213-223.

[27] J. C. Brown, M. Haverkorn, B. M. Gaensler, A. R. Taylor, N. S. Bizunok, N. M. McClure-Griffiths, J. M. Dickey and A. J. Green, Rotation measures of extragalactic sources behind the southern Galactic plane: New insights into the large-scale magnetic field of the inner Milky Way, ApJ $66 \mathbf{6}$ (2007) 258-266.

[28] J. C. Brown and A. R. Taylor, The Structure of the Magnetic Field in the Outer Galaxy from Rotation Measure Observations through the Disk, ApJ 563 (Dec., 2001) L31-L34.

[29] B. M. Gaensler, M. Haverkorn, L. Staveley-Smith, J. M. Dickey, N. M. McClure-Griffiths, J. R. Dickel and M. Wolleben, The magnetic field of the Large Magellanic Cloud revealed through Faraday rotation, Science 307 (2005) 1610-1612.

[30] M. Hanasz, G. Kowal, K. Otmianowska-Mazur and H. Lesch, Amplification of galactic magnetic fields by the cosmic-ray-driven dynamo, ApJ 605 (2004) L33-L36.

[31] R. Beck and B. M. Gaensler, Observations of magnetic fields in the Milky Way and in nearby galaxies with a Square Kilometre Array, New Astron. Rev. 48 (2004) 1289-1304.

[32] B. M. Gaensler, R. Beck and L. Feretti, The origin and evolution of cosmic magnetism, New Astron. Rev. 48 (2004) 1003-1012.

[33] A. R. Taylor, J. M. Stil, J. K. Grant, T. L. Landecker, R. Kothes, R. Reid, A. D. Gray, D. Scott, P. G. Martin, A. Boothroyd, G. Joncas, F. J. Lockman, J. English, A. Sajina and J. R. Bond, Radio polarimetry of the ELAIS N1 field: Polarized compact sources, ApJ 666 (2007) 201-211. 
[34] R. Stepanov, T. G. Arshakian, R. Beck, P. Frick and M. Krause, Magnetic field structures of galaxies derived from analysis of Faraday rotation measures, and perspectives for the SKA, A\&A (2008), in press (arXiv:0711.1267).

[35] T. Kolatt, Determination of the primordial magnetic field power spectrum by faraday rotation correlations, ApJ 495 (1998) 564-579.

[36] P. Blasi, S. Burles and A. V. Olinto, Cosmological magnetic field limits in an inhomogeneous universe, ApJ 514 (1999) L79-L82.

[37] P. P. Kronberg, M. L. Bernet, F. Miniati, S. J. Lilly, M. B. Short and D. M. Higdon, A global probe of cosmic magnetic fields to high redshifts, ApJ (2008), in press arXiv:0712.0435.

[38] P. P. Kronberg, J. J. Perry and E. L. H. Zukowski, Discovery of extended Faraday rotation compatible with spiral structure in an intervening galaxy at $z=0.395$ : New observations of PKS 1229-021, ApJ 387 (1992) 528-535.

[39] D. A. Frail, P. B. Cameron, M. Kasliwal, E. Nakar, P. A. Price, E. Berger, A. Gal-Yam, S. R. Kulkarni, D. B. Fox, A. M. Soderberg, B. P. Schmidt, E. Ofek and S. B. Cenko, An energetic afterglow from a distant stellar explosion, ApJ 646 (2006) L99-L102.

[40] I. D. McGreer, R. H. Becker, D. J. Helfand and R. L. White, Discovery of a $z=6.1$ radio-loud quasar in the NOAO Deep Wide Field Survey, AJ 652 (2006) 157-162.

[41] A. Kosowsky and A. Loeb, Faraday rotation of microwave background polarization by a primordial magnetic field, ApJ 469 (1996) 1-6.

[42] C. Scóccola, D. Harari and S. Mollerach, B polarization of the CMB from Faraday rotation, Phys. Rev. D 70 (2004) 063003.

[43] T. A. Enßlin, A. Waelkens, C. Vogt and A. A. Schekochihin, Future magnetic field studies using the Planck surveyor experiment, Astron. Nach. 327 (2006) 626-631.

[44] A. De Angelis, M. Persic and M. Roncadelli, Constraints on large-scale magnetic fields from the Auger results, preprint, arXiv:0711.3346.

[45] H. J. A. Röttgering, B. R., P. D. Barthel, M. P. van Haarlem, G. K. Miley, G. K. Morganti and I. Snellen, LOFAR - Opening up a new window on the Universe, in Cosmology, Galaxy Formation

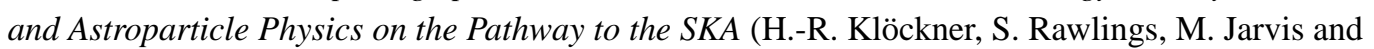
A. Taylor, eds.), 2006

[46] S. Johnston, et al., Science with the Australian Square Kilometre Array Pathfinder, PASA 24 (2007) 174-188. 